\title{
El aumento de peso durante el embarazo se correlaciona con el peso del recién nacido
}

\author{
Weight gain during pregnancy is associated with birthweight
}

Ludwig DS y col. Lancet 2010;376:984-90.

\section{Objetivos}

Evaluar la asociación entre ganancia de peso materno y peso al nacimiento comparando distintos embarazos en la misma madre.

\section{Diseño}

Estudio de cohortes de base poblacional. Tomando en cuenta todos los nacimientos ocurridos en Michigan y New Jersey (EE.UU.), entre 1989 y 2003, se seleccionaron aquellos gestados a partir de embarazos únicos en madres que tuvieron más de un parto. Se excluyeron las gestaciones de menos de 37 o mas de 41 semanas, y aquellas con peso al nacimiento $<500$ o > $7000 \mathrm{~g}$; también a las madres con diabetes y aquellas en las cuales no hubieren adecuados registros. Se incluyeron 513.501 mujeres y sus 1.164 .750 recién nacidos $(\mathrm{RN})$.

\section{Evaluación de factores de riesgo}

Se evaluaron las variables: ganancia de peso durante el embarazo, peso al nacimiento, diabetes gestacional, semanas de gestación, edad materna, educación materna, estado civil de la madre, grupo étnico, tabaquismo, control prenatal adecuado, modo de nacimiento, sexo del RN, y año de nacimiento.

\section{Resultados principales}

Se evaluó la asociación entre la ganancia de peso materno en dos o más embarazos y el peso del RN. Se reporta una relación lineal estimando un aumento de $7,35 \mathrm{~g}$ en el peso del RN por cada kilo de aumento de peso de la madre (IC 95\% 7,1 a 7,59 $\mathrm{p}<0,0001)$.

Para reducir los confundidores posibles, se utilizó un tipo especial de modelo de regresión lineal múltiple* y se confirmaron los hallazgos restringiendo el análisis a los subgrupos específicos pre-establecidos en base a los factores de riesgo más importantes (edad gestacional entre 39 y 40 semanas, no fumadoras, parto vaginal, y control prenatal adecuado).

Los $\mathrm{RN}$ de las pacientes que aumentaron más de $24 \mathrm{~kg}$ durante el embarazo fueron $148,9 \mathrm{~g}$ (141,7 a 156) más pesados que los $\mathrm{RN}$ de las mujeres que aumentaron entre 8 a10 kg (grupo de referencia) y el odds ratio* de tener un $\mathrm{RN}$ con un peso $>4.000$ gr. fue 2,26 (IC95\% 2,09 a 2,44).

\section{Conclusiones}

El aumento de peso materno durante el embarazo se correlaciona con el aumento el peso del RN independientemente de la influencia de factores genéticos.

Palabras claves: macrosomía fetal, ganancia de peso materno, peso al nacimiento Key words: fetal macrosomia, maternal weight gain, birth weight. Fuente de financiamiento: US National Institutes of Health.

\section{Comentario}

El aumento excesivo de peso durante el embarazo ha sido asociado a complicaciones maternas y fetales con posibles repercusiones a largo plazo'. El alto peso al nacimiento está relacionado con un mayor riesgo de nacimientos por cesárea, desgarros del canal del parto, hemorragias postparto y otras complicaciones para la madre, y de distocia de hombros y otros traumatismos para el $\mathrm{RN}^{2,3}$.

La ganancia de peso durante el embarazo recomendada por el Instituto de Medicina ${ }^{4}$ (en inglés, IOM) depende del índice de masa corporal (IMC) de la mujer antes del embarazo: para mujeres con un IMC bajo $\left(<18,5 \mathrm{~kg} / \mathrm{m}^{2}\right)$, entre 12,7 y $18 \mathrm{~kg}$; en aquellas con un peso normal (IMC 18,5 a 24,9 kg/m²), entre 11,3 y 15,9 kg; en aquellas con sobrepeso ( $>25$ a $29,9 \mathrm{~kg} / \mathrm{m}^{2}$ ) entre 6,8 y $11,3 \mathrm{~kg}$; y en obesas (IMC >30 kg/m²), entre 5 a $9 \mathrm{~kg}$.

En una revisión sistemática de 35 estudios, Siega-Riz y colaboradores evaluaron las consecuencias de la ganancia de peso materno, concluyendo que una ganancia de peso menor a la recomendada se asociaba a $\mathrm{RN}$ de bajo peso, mientras que la ganancia excesiva se asociaba con $\mathrm{RN}$ de mayor peso al nacimiento, crecimiento fetal acelerado y mayor retención de peso postparto ${ }^{5}$.

En este trabajo, los autores utilizaron los certificados de nacimiento de más de medio millón de mujeres para evaluar la relación entre la ganancia de peso materno y el peso del RN, encontrando correlación positiva entre aumento durante el embarazo y el peso del RN, así como correlación intra-sujeto, al aparear los pesos de distintos embarazos de la misma madre. Sin embargo, por utilizar datos de registros vitales no permite controlar todos los potenciales confundidores.

\section{Conclusiones de los comentadores}

El control prenatal brinda una oportunidad única para ayudar a las embarazadas a evitar una ganancia de peso excesiva, y de esta manera prevenir resultados perinatales adversos.

María Laura Giménez [ Servicio de Obstetricia del Hospital Italiano de Buenos Aires. marial.gimenez@hospitalitaliano.org.ar ]

*Ver glosario Gustavo Izbizky [ Subjefe del Servicio de Obstetricia del Hospital Italiano de Buenos Aires. gustavo.izbizky @ hospitalitaliano.org.ar ]

Gimenez ML, Izbizky G. El aumento de peso durante el embarazo se correlaciona con el peso del recién nacido. Evid Act Pract Ambul. 14(2). 47. Comentado de: Ludwig DS, Currie J. The association between pregnancy weight gain and birthweight: a within-family comparison. Lancet 2010;376:984-90. PMID: 20691469.

\section{Referencias}

1. Artal R y col. Weight gain recommendations in pregnancy and the obesity epidemic. Obstet Gynecol 2010;115(1):152-5.

2. Ferber A. Maternal complications of fetal macrosomia. Clin Obstet Gynecol 2000;43:335-39.

3. Grassi AE, Giuliano MA.The neonate with macrosomia. Clin Obstet Gynecol 2000;43:340-48

4. Institute of Medicine. Committee to reexamine IOM pregnancy weight guidelines. In: Rasmussen KM, Yaktine AL, eds. Weight gain during pregnancy: reexamining the guidelines. 2009.

5. Siega-Riz AM y col. A systematic review of outcomes of maternal weight gain according to the Institute of Medicine recommendations: birthweight, fetal growth, and postpartum weight retention. Am J Obstet Gynecol 2009;201(4):339.e1-14. 